\title{
ON THE DIFFERENCE OF VALUES OF THE KERNEL FUNCTION AT CONSECUTIVE INTEGERS
}

\author{
JEAN-MARIE DE KONINCK and FLORIAN LUCA
}

Received 1 April 2003

\begin{abstract}
For each positive integer $n$, set $\gamma(n)=\prod_{p \mid n} p$. Given a fixed integer $k \neq \pm 1$, we establish that if the $A B C$-conjecture holds, then the equation $\gamma(n+1)-\gamma(n)=k$ has only finitely many solutions. In the particular cases $k= \pm 1$, we provide a large family of solutions for each of the corresponding equations.
\end{abstract}

2000 Mathematics Subject Classification: 11A25.

1. Introduction. Comparing values of an arithmetic function at consecutive integers is a common problem in number theory. For example, in 1952, Erdős and Mirsky [4] asked if there are infinitely many integers $n$ such that $d(n)=$ $d(n+1)$ (here $d(n)$ stands for the number of divisors of $n$ ), a question which was answered in the affirmative when Heath-Brown [8] proved in 1984 that the number of positive integers $n \leq x$ such that $d(n)=d(n+1)$ is $\gg x / \log ^{7} x$, a lower bound which was later improved by Hildebrand [9] and thereafter by Erdős et al. [6].

An apparently more difficult problem seems to be that of establishing that the equations $\phi(n)=\phi(n+1)$ (where $\phi$ is Euler's function) and $\sigma(n)=$ $\sigma(n+1)$ (where $\sigma(n)$ stands for the sum of the divisors of $n$ ) each has infinitely many solutions $n$; see Erdős et al. [5] for developments concerning this problem.

The distribution of the values of the kernel function $\gamma(n):=\prod_{p \mid n} p$ (also called the core function or the algebraic radical of $n$ ) is the source of a variety of open problems, many of them tied in with the famous $A B C$-conjecture. For instance, in 1999, Cochrane and Dressler [2] showed that, assuming the $A B C$ conjecture, if two positive integers have the same prime factors, they cannot be too close; more precisely, they proved that if the $A B C$-conjecture is true, then given any $\varepsilon>0$, there exists a positive constant $C=C(\varepsilon)$ such that if $\gamma(n)=\gamma(n+k)$, then $k \geq C n^{1 / 2-\varepsilon}$. No easier is the conjecture of P. Erdós and A. Woods which asserts that there exists an integer $k \geq 3$ such that if $m$ and $n$ are positive integers satisfying $\gamma(m+i)=\gamma(n+i)$ for all $1 \leq i \leq k$, then $m=n$. Although it remains unsolved, this conjecture has been extensively studied and generalized; see, for instance, Langevin [10], Balasubramanian et al. [1], as well as Langevin [11]. 
Many more results regarding the kernel function and the $A B C$-conjecture have been published; for some recent ones, see Mitrinović et al. [12], Granville [7], or Cutter et al. [3].

Perhaps an even more difficult problem seems to be the one of comparing the values of the kernel function at consecutive integers. In this note, we look at the values of the function $\gamma(n+1)-\gamma(n)$ for positive integers $n$.

2. Preliminary observations and statement of the main results. We first make a few observations. Note that it is always the case that one of the numbers $n$ and $n+1$ is even and the other one is odd. In particular, $\gamma(n+1)-\gamma(n)$ is always an odd number, and therefore the equation $\gamma(n+1)-\gamma(n)=k$ has no solutions when $k$ is a fixed even positive integer. From now on, we assume that $k$ is a fixed odd positive integer. When $k=1$ and both $n$ and $n+1$ are square-free, we certainly have that $\gamma(n+1)-\gamma(n)=(n+1)-n=1=k$. Since, for a large positive real number $x$, there are only $\left(1-6 / \pi^{2}+o(1)\right) x$ positive integers $n<x$ for which $n$ is not square-free, it follows that there are at most $\left(2-12 / \pi^{2}+o(1)\right) x$ positive integers $n<x$ such that one of $n$ or $n+1$ is not square-free. In particular, the number of numbers $n<x$ for which both $n$ and $n+1$ are square-free is at least

$$
x-\left(2-\frac{12}{\pi^{2}}+o(1)\right) x=\left(\frac{12}{\pi^{2}}-1+o(1)\right) x>0.215 x
$$

Thus, the solutions $n$ of the equation $\gamma(n+1)-\gamma(n)=1$ form a subset of all the positive integers of positive lower asymptotic density.

From now on, we look for positive integer solutions $n$ of the equation $\gamma(n+$ $1)-\gamma(n)=k$ such that $n(n+1)$ is not square-free. Here is a "parametric family" of solutions for $k=1$. Let $r>1$ be an integer and assume that both $2^{r-1}-1$ and $2^{r}-1$ are square-free. Put $n=2^{r+1}\left(2^{r-1}-1\right)$. Then $n+1=$ $2^{r+1}\left(2^{r-1}-1\right)+1=2^{2 r}-2^{r+1}+1=\left(2^{r}-1\right)^{2}$. It is clear that $n+1$ is not squarefree, and if $r \geq 3$, then $n$ is not square-free either. Since both $2^{r-1}-1$ and $2^{r}-1$ are square-free, we have $\gamma(n+1)=2^{r}-1, \gamma(n)=2\left(2^{r-1}-1\right)=2^{r}-2$, which implies that $\gamma(n+1)-\gamma(n)=\left(2^{r}-1\right)-\left(2^{r}-2\right)=1$. It is not even known if there are infinitely many $r$ such that $2^{r}-1$ is square-free, and therefore it is not known if there are infinitely many solutions $n$ of the above form to the equation $\gamma(n+1)-\gamma(n)=1$. However, computations revealed that there are 106 values of the positive integer $r<200$ having the property that both $2^{r-1}-1$ and $2^{r}-1$ are square-free; all these values are listed in Section 5. A similar type of "parametric solution" can be found when $k=-1$. In this case, if $r>1$ is such that both $2^{r-1}+1$ and $2^{r}+1$ are square-free, then taking $n=2^{r+1}\left(2^{r-1}+1\right)$, we have $n+1=\left(2^{r}+1\right)^{2}$, in which case $\gamma(n+1)-\gamma(n)=\left(2^{r}+1\right)-2\left(2^{r-1}+1\right)=-1$. The list of those $r<200$ such that both $2^{r-1}+1$ and $2^{r}+1$ are square-free is also included in Section 5 . 
We conjecture that for every fixed value of $k \neq \pm 1$, the equation $\gamma(n+1)-$ $\gamma(n)=k$ has only finitely many positive integer solutions $n$; the solutions $n<10^{9}$ of this equation, when $1<|k|<100$, are given in Section 4 . We also conjecture that when $k= \pm 1$, the equation $\gamma(n+1)-\gamma(n)=k$ has only finitely many positive integer solutions $n$, which are not of one of the forms specified above.

In this note, we prove that our conjectures are implied by the $A B C$-conjecture. In fact, assuming the $A B C$-conjecture, we prove a much stronger statement which implies the above conjectures.

We first recall that the $A B C$-conjecture is the following statement.

The $A B C$-Conjecture. For every $\varepsilon>0$, there exists a constant $K:=K(\varepsilon)$ such that whenever $A, B, C$ are three coprime nonzero integers with $A+B=C$, then

$$
\max \{|A|,|B|,|C|\}<K \gamma(A B C)^{1+\varepsilon} .
$$

We will choose to write the above inequality as

$$
\max \{|A|,|B|,|C|\} \ll_{\varepsilon} \gamma(A B C)^{1+\varepsilon} .
$$

THEOREM 2.1. (i) Let $\varepsilon>0$ be given. Then the $A B C$-conjecture implies that the inequality

$$
|\gamma(n+1)-\gamma(n)| \gg_{\varepsilon} n^{1 / 10-\varepsilon}
$$

holds whenever $|\gamma(n+1)-\gamma(n)|>1$. In particular, if $k>1$ is any fixed positive integer, then the equation $|\gamma(n+1)-\gamma(n)|=k$ has only finitely positive integer solutions $n$.

(ii) The ABC-conjecture implies that there are only finitely many positive solutions $n$ to the equation $\gamma(n+1)-\gamma(n)=1$ such that $n(n+1)$ is not square-free, and such that $n$ is not of the form $n=2^{r+1}\left(2^{r-1}-1\right)$ with some integer $r>1$ such that both $2^{r-1}-1$ and $2^{r}-1$ are square-free.

(iii) The $A B C$-conjecture implies that there are only finitely many positive solutions $n$ to the equation $\gamma(n+1)-\gamma(n)=-1$ for which $n$ is not of the form $n=2^{r+1}\left(2^{r-1}+1\right)$ with some integer $r>1$ such that both $2^{r-1}+1$ and $2^{r}+1$ are square-free.

REMARK 2.2. The above result implies that the only cluster points of the sequence $\{|\gamma(n+1)-\gamma(n)|\}_{n \geq 1}$ are 1 and infinity, and that

$$
\liminf _{\substack{n \rightarrow \infty \\ n \in \mathscr{A}}} \frac{\log |\gamma(n+1)-\gamma(n)|}{\log n} \geq \frac{1}{10},
$$


where $\mathscr{A}$ is the set of all positive integers $n$ such that $n(n+1)$ is not squarefree, and $n$ is not of the form $2^{r+1}\left(2^{r-1} \pm 1\right)$.

The following result is a more general version of Theorem 2.1.

THEOREM 2.3. (i) Let $\varepsilon>0$ be given. Then the $A B C$-conjecture implies that the inequality

$$
|\gamma(m)-\gamma(n)| \gg_{\varepsilon}|m-n|^{1 / 15-\varepsilon}
$$

holds for all coprime positive integers $m$ and $n$.

(ii) Let $\varepsilon>0$ be given and let $j>1$ be a fixed integer. Then the $A B C$-conjecture implies that the inequality

$$
|\gamma(n+j)-\gamma(n)| \gg_{j, \varepsilon} n^{1 / 10-\varepsilon}
$$

holds for all positive integers $n$ coprime to $j$ such that $|\gamma(n+j)-\gamma(n)|>j$.

(iii) Let $j>1$ be a fixed integer. Then the ABC-conjecture implies that all but finitely many solutions in positive integers $n$ coprime to $j$ of the inequality

$$
|\gamma(n+j)-\gamma(n)| \leq j
$$

have the property that $n(n+j)$ is square-free, unless $j=j_{0}^{2}$ is a perfect square, in which case all the other solutions of inequality (2.8) are of the form $n=$ $2^{r}\left(2^{r}+2 \eta j_{0}\right)$, for some $\eta \in\{ \pm 1\}$ and some nonnegative integer $r$ such that both $2^{r}+2 \eta j_{0}$ and $2^{r}+\eta j_{0}$ are square-free.

3. The proof of Theorem 2.1. We let $\varepsilon>0$ be some small number. Now let $k$ be an odd integer and $n$ a positive integer such that $\gamma(n+1)-\gamma(n)=k$. Furthermore, let $a$ and $b$ be the two square-free integers given by $a:=\gamma(n+$ $1)$ and $b:=\gamma(n)$. Assume first that $\max \{a, b\} \leq 2|k|$. In this case, the $A B C$ conjecture applied to the equation

$$
(n+1)-n=1
$$

yields

$$
n \leq n+1 \ll_{\varepsilon}(\gamma(n) \gamma(n+1))^{1+\varepsilon} \ll_{\varepsilon}(a b)^{1+\varepsilon} \ll_{\varepsilon}(2|k|)^{2(1+\varepsilon)} \ll_{\varepsilon}|k|^{2(1+\varepsilon)},
$$

leading to

$$
|k| \gg_{\varepsilon} n^{1 / 2(1+\varepsilon)}
$$


which is an even better inequality than inequality (2.4). We note that when $k$ is fixed, then the fact that the equation $\gamma(n+1)-\gamma(n)=k$ has only finitely many positive integer solutions $n$ satisfying $\max \{a, b\} \leq 2|k|$ can be proved unconditionally as follows. Let $\mathscr{P}$ be the set of all prime numbers $p \leq 2|k|$ and let $\mathscr{S}$ be the set of all positive integers whose prime factors belong to $\mathscr{P}$. In this case, both $n$ and $n+1$ belong to $\mathscr{Y}$, and therefore the pair $(x, y):=(n+1, n)$ is a solution of the equation $x-y=1$, with $x, y \in \mathscr{Y}$, and it is known that such a diophantine equation has only finitely many solutions $(x, y)$ which are effectively computable.

Thus, we may assume that $\max \{a, b\}>2|k|$. In this case, since $a-b=k$, it follows that $\max \{a, b\}<2 \min \{a, b\}$. In particular, both inequalities $a<2 b$ and $b<2 a$ hold. Further, let $c:=(n+1) / \gamma(n+1)$ and $d:=n / \gamma(n)$. We may assume that $\max \{c, d\}>1$, for otherwise both $n$ and $n+1$ are square-free, and this implies that $k=1$. We now have the system of equations

$$
a-b=k, \quad c a-d b=1 .
$$

Applying the $A B C$-conjecture to the second equation of (3.4), we get

$$
c a \ll_{\varepsilon} \gamma(a b c d)^{1+\varepsilon}=(a b)^{1+\varepsilon} \ll_{\varepsilon}\left(2 a^{2}\right)^{1+\varepsilon} \ll_{\varepsilon} a^{2+2 \varepsilon} .
$$

Inequality (3.5) implies that

$$
c \ll_{\varepsilon} a^{1+2 \varepsilon} \ll_{\varepsilon}(2 b)^{1+2 \varepsilon} \ll_{\varepsilon} b^{1+2 \varepsilon} .
$$

A similar argument shows that the inequality

$$
d \ll_{\varepsilon}(\min \{a, b\})^{1+2 \varepsilon}
$$

holds. We now multiply both sides of the first equation of (3.4) by $c$ and subtract the second equation of (3.4) to get

$$
k c-1=d b-c b=b(d-c) .
$$

Note that $d$ and $c$ are coprimes and that at least one of them is larger than 1 . Hence, $d-c \neq 0$. Thus, in view of (3.6) and (3.8),

$$
|d-c| b=|k c-1| \leq 2|k| c \ll_{\varepsilon}|k| b^{1+2 \varepsilon}
$$

so that

$$
|d-c| \ll_{\varepsilon}|k| b^{2 \varepsilon}
$$


In particular, since $b<2 a$, it follows that

$$
|d-c| \ll|k|(\min \{a, b\})^{2 \varepsilon} .
$$

We now write $d-c=e$. Then, since $b=a-k$ and $d=c+e$, we have

$$
1=c a-d b=c a-(c+e)(a-k)=c a-(c a+e a-k c-k e)=k c-e a+k e,
$$

in which case

$$
k c-e a=1-k e .
$$

Assume first that $1-k e \neq 0$. In this case, since $\gamma(c) \mid a$, we read from (3.13) that $\gamma(c)$ divides $|1-k e|$. In particular, it follows from (3.11) and (3.13) that

$$
\gamma(c) \leq|1-k e| \leq 2|k||e| \ll_{\varepsilon}|k|^{2} a^{2 \varepsilon} .
$$

Therefore,

$$
1=c a-d b=(d-e)(b+k)-d b=(d b-e b+k d-e k)-d b=-e b+k d-e k,
$$

and hence,

$$
k d-e b=1+e k .
$$

Assume now that $1+e k \neq 0$. In this case, since $\gamma(d) \mid b$, we get that $\gamma(d)$ divides $|1+e k|$ and therefore

$$
\gamma(d) \leq|1+e k| \leq 2|k||e| \ll_{\varepsilon}|k|^{2} a^{2 \varepsilon} .
$$

Applying the $A B C$-conjecture to the equation

$$
d-c=e,
$$

we get, using (3.11), (3.14), and (3.17), that

$$
\begin{aligned}
\max \{d, c\} & \ll_{\varepsilon} \gamma(d c|e|)^{1+\varepsilon} \ll_{\varepsilon}(\gamma(d) \gamma(c)|e|)^{1+\varepsilon} \\
& \ll_{\varepsilon}\left(|k|^{4} a^{4 \varepsilon}|e|\right)^{1+\varepsilon} \ll|k|^{4(1+\varepsilon)} a^{5 \varepsilon}|e|^{1+\varepsilon},
\end{aligned}
$$


provided that $\varepsilon<1 / 4$. On the one hand, inequality (3.19) combined with (3.11) gives

$$
\max \{d, c\} \ll_{\varepsilon}|k|^{4(1+\varepsilon)} a^{5 \varepsilon}|e|^{1+\varepsilon} \ll_{\varepsilon}|k|^{5(1+\varepsilon)} a^{\varepsilon(5+2(1+\varepsilon))} \ll_{\varepsilon}|k|^{5(1+\varepsilon)} a^{8 \varepsilon},
$$

provided that $\varepsilon<1 / 2$, while on the other hand, returning to (3.8), it follows from (3.19) and (3.11) that

$$
b|e|=b|d-c|=|k c-1| \leq 2|k| c \ll_{\varepsilon}|k|^{5+4 \varepsilon} a^{5 \varepsilon}|e|^{1+\varepsilon},
$$

and therefore

$$
b \ll_{\varepsilon}|k|^{5+4 \varepsilon} a^{5 \varepsilon}|e|^{\varepsilon} \ll_{\varepsilon}|k|^{5(1+\varepsilon)} a^{7 \varepsilon} .
$$

Since $a \leq 2 b$, it follows from (3.22) that

$$
b \ll_{\varepsilon}|k|^{5(1+\varepsilon) /(1-7 \varepsilon)} \ll_{\varepsilon}|k|^{5(1+10 \varepsilon)},
$$

provided that $\varepsilon<1 / 35$. Substituting (3.23) into (3.20) and using again the fact that $a \leq 2 b$, we get

$$
\max \{d, c\} \ll_{\varepsilon}|k|^{5((1+\varepsilon)+8 \varepsilon(1+10 \varepsilon))} \ll_{\varepsilon}|k|^{5(1+10 \varepsilon)},
$$

provided that $\varepsilon<1 / 80$. From (3.23) and (3.24), we immediately get that

$$
n=b d \ll_{\varepsilon}|k|^{10(1+10 \varepsilon)},
$$

leading to

$$
|k| \gg_{\varepsilon} n^{1 / 10(1+10 \varepsilon)} \gg_{\varepsilon} n^{1 / 10-\varepsilon},
$$

which is precisely inequality (2.4).

Our reasoning was based on the fact that we assumed, aside from the $A B C$ conjecture, that $1-k e \neq 0$ and $1+k e \neq 0$. Hence, we now assume that (1$k e)(1+k e)=0$. Note that this is possible only when $|k|=1$, which, together with the previous arguments, justifies Theorem 2.1(i). Now assume that $1-$ $k e=0$. In this case, $k e=1$ and therefore $1+k e=2$. Equation (3.16) now tells us that $\gamma(d) \mid 2$ and therefore $d=2^{r}$ for some integer $r \geq 0$. Since $k e=1$, we either have $k=e=1$ or $k=e=-1$. When $k=e=1$, we have $d-c=1$, in which case $c=d-1=2^{r}-1$ and $a-b=1$. From (3.13), we also have $c-e a=0$ so that $a=e a=c=2^{r}-1$, and $b=a-1=2^{r}-2=2\left(2^{r-1}-1\right)$. The condition that $a$ and $b$ are positive and square-free forces $r>1$ and both $2^{r}-1$ and $2^{r-1}-1$ to be square-free. Hence $n+1=a c=\left(2^{r}-1\right)^{2}$, while $n=\left(2^{r}-1\right)^{2}-1=$ $2^{r+1}\left(2^{r-1}-1\right)$, which is exactly the parametric family mentioned in Section 2. Now assume that $k=e=-1$. In this case, we have $d-c=-1$, which implies 
that $c=d+1=2^{r}+1$, and $a-b=-1$. From (3.13), we also have $c=a$ so that $a=2^{r}+1$, and $b=a+1=2^{r}+2=2\left(2^{r-1}+1\right)$. The condition that $a$ and $b$ are square-free forces $r>1$ and $2^{r-1}+1$ and $2^{r}+1$ to be square-free. Thus $n+1=\left(2^{r}+1\right)^{2}$ and $n=\left(2^{r}+1\right)^{2}-1=2^{r+1}\left(2^{r-1}+1\right)$. Now assume that $1+k e=0$, in which case (3.16) shows that $k d=e b$. Since $k e=-1$, we get that $k=-e \in\{ \pm 1\}$, and therefore $d=-b$. This is impossible because both $d$ and $b$ are positive. The proof of Theorem 2.1 is thus complete.

4. The proof of Theorem 2.3. The proof of this result can be achieved by following the same procedure as in the proof of Theorem 2.1, and we will only sketch it. Let $\varepsilon>0$ be a very small number. Put $j:=m-n, k:=\gamma(m)-\gamma(n)$, and $K:=\max \{j,|k|\}$. We may assume that $j>|k|$, for otherwise we already have that $|\gamma(m)-\gamma(n)|=|k| \geq j=|m-n|$, which implies inequality (2.6).

We write $a:=\gamma(m)$ and $b:=\gamma(n)$. If $\max \{a, b\} \leq 2 K$, then the $A B C$ conjecture applied to the equation $m-n=j$ shows that

$$
j \ll_{\varepsilon}(a b K)^{1+\varepsilon} \ll_{\varepsilon} K^{3(1+\varepsilon)},
$$

which gives

$$
K \gg_{\varepsilon} j^{1 / 3(1+\varepsilon)},
$$

which is a better inequality than the one asserted at (2.6). Thus, we may assume that $\max \{a, b\}>2 K$. As in the proof of Theorem 2.1, we set $c:=m / \gamma(m)$ and $d:=n / \gamma(n)$ and we have the system of equations

$$
a-b=k, \quad c a-d b=j .
$$

Applying the $A B C$-conjecture to the second equation of (4.3), we get

$$
c a \ll_{\varepsilon}(\gamma(a b c d) j)^{1+\varepsilon} \ll_{\varepsilon} K^{1+\varepsilon} a^{2+2 \varepsilon},
$$

which, together with the fact that $a \leq 2 b$, leads easily to the fact that

$$
c \ll_{\varepsilon} K^{1+\varepsilon}(\min \{a, b\})^{1+2 \varepsilon} .
$$

In the same way, one shows that

$$
d \ll_{\varepsilon} K^{1+\varepsilon}(\min \{a, b\})^{1+2 \varepsilon} .
$$

We now multiply both sides of the first equation of (4.3) by $c$ and subtract the second equation of (4.3) to get

$$
k c-j=b(d-c) .
$$

Note that $d$ and $c$ are coprimes, thus $d-c=0$ only when $d=c=1$. This, in turn, is possible only when both $m$ and $n$ are square-free, therefore $|k|=j$, 
which is a contradiction. Hence, $d-c \neq 0$. Thus, in view of (4.5) and of (4.7),

$$
b|d-c|=|k c-j| \leq K c \ll_{\varepsilon} K^{2+\varepsilon} b^{1+2 \varepsilon}
$$

so that

$$
|d-c| \ll_{\varepsilon} K^{2+\varepsilon} b^{2 \varepsilon},
$$

and since $b \leq 2 a$, we get that

$$
|d-c| \ll_{\varepsilon} K^{2+\varepsilon}(\min \{a, b\})^{2 \varepsilon} .
$$

As before, we let $e=d-c$ and using the fact that $b=a-k$ and $d=c+e$, we rewrite the second equation of (4.3) as

$$
k c-e a=j-k e .
$$

Assume that $j-k e \neq 0$. Since $\gamma(c) \mid a$, we then get from (4.11) and (4.10) that

$$
\gamma(c) \leq|j-k e| \ll K|e| \ll_{\varepsilon} K^{3+\varepsilon}(\min \{a, b\})^{2 \varepsilon} .
$$

An inequality similar to (4.12) holds with $c$ replaced by $d$ provided that $j+k e \neq$ 0 , and now the $A B C$-conjecture applied to the equation $d-c=e$ gives

$$
\begin{aligned}
\max \{c, d\} & \ll_{\varepsilon}(\gamma(c) \gamma(d)|e|)^{1+\varepsilon} \ll_{\varepsilon} K^{(6+2 \varepsilon)(1+\varepsilon)}(\min \{a, b\})^{4 \varepsilon(1+\varepsilon)}|e|^{1+\varepsilon} \\
& \ll_{\varepsilon} K^{6(1+2 \varepsilon)}(\min \{a, b\})^{5 \varepsilon}|e|^{1+\varepsilon},
\end{aligned}
$$

provided that $\varepsilon<1 / 4$. On the one hand, inequality (4.13), together with (4.10), gives

$$
\begin{aligned}
\max \{c, d\} & \ll_{\varepsilon} K^{6(1+2 \varepsilon)+(2+\varepsilon)(1+\varepsilon)}(\min \{a, b\})^{5 \varepsilon+2 \varepsilon(1+\varepsilon)} \\
& \ll_{\varepsilon} K^{8(1+2 \varepsilon)}(\min \{a, b\})^{8 \varepsilon}
\end{aligned}
$$

provided that $\varepsilon<1 / 2$, while, on the other hand, inequality (4.13) and (4.7) show that

$$
b|e|=|k c-j| \leq K c \ll_{\varepsilon} K^{7+12 \varepsilon}(\min \{a, b\})^{5 \varepsilon}|e|^{1+\varepsilon}
$$

and therefore

$$
\begin{aligned}
b & \ll_{\varepsilon} K^{7+12 \varepsilon}(\min \{a, b\})^{5 \varepsilon}|e|^{\varepsilon} \\
& \ll_{\varepsilon} K^{7+12 \varepsilon+\varepsilon(2+\varepsilon)}(\min \{a, b\})^{5 \varepsilon+2 \varepsilon^{2}} \ll_{\varepsilon} K^{7+15 \varepsilon}(\min \{a, b\})^{7 \varepsilon} .
\end{aligned}
$$

Multiplying (4.14) and (4.16) and using the fact that $a \leq m$, we get

$$
m=b d \ll_{\varepsilon} K^{15+31 \varepsilon} m^{15 \varepsilon},
$$


leading to the conclusion that

$$
K \gg_{\varepsilon} m^{(1-15 \varepsilon) /(15+31 \varepsilon)} \gg_{\varepsilon} m^{1 / 15-2 \varepsilon} \gg_{\varepsilon} j^{1 / 15-2 \varepsilon},
$$

which implies inequality (2.6) in light of the fact that $\varepsilon$ can be taken arbitrarily small.

It remains to consider the degenerate cases in which $j \pm k e=0$. Assume first that $j-k e=0$. In this case, we have that $k \mid j$. Put $j=k j_{0}$. Then $e=j_{0}$. Note that $k$ and $j_{0}$ have the same sign. Equation (4.11) then shows that $k c=$ $e a=j_{0} a$. Write $D:=\operatorname{gcd}\left(k, j_{0}\right)>0$ and write $k=D k_{1}, j_{0}=D j_{1}$. Note that $k_{1}$ and $j_{1}$ have the same sign. We then get that $c=j_{1} \rho$ and $a=k_{1} \rho$, and since $\gamma(c) \mid a$ and $a$ is square-free, we get that $j_{1} \mid \rho$, and therefore $\rho=j_{1} \rho_{1}$, which implies that $c=j_{1}^{2} \rho_{1}$ and $a=k_{1} j_{1} \rho_{1}$. The analogue of relation (3.16) is now $k d-e b=j+e k=2 j$, which can be rewritten as $k d-j_{0} b=2 k j_{0}$. Simplifying $D$, we get $k_{1} d-j_{1} b=2 k_{1} j_{0}$. Reducing this modulo $k_{1}$, we get that $k_{1}$ divides $j_{1} b$, and since $k_{1}$ is coprime to $j_{1}$, we get that $k_{1}$ divides $b$. But $k_{1}$ also divides $a$, therefore $k_{1}$ divides both $m$ and $n$, which shows that $k_{1}= \pm 1$. Since $j_{0}$ is a multiple of $j_{1}$, we may reduce the above equation modulo $j_{1}$ and read that $j_{1}$ divides $k_{1} d$, which implies that $j_{1}$ divides $d$. Since it also divides $a$, it follows that $j_{1}= \pm 1$. Thus, we have showed that $k=\eta j_{0}, j=j_{0}^{2}, a=c$, and $d=b+2 \eta j_{0}$, where $\eta \in\{ \pm 1\}$. The relation $a-b=k$ gives $a=b+\eta j_{0}$, therefore $m=a c=\left(b+\eta j_{0}\right)^{2}$ and $n=b d=b\left(b+2 \eta j_{0}\right)$. It is clear that $m-n=j$, and the only restriction now is that both $b$ and $b+\eta j_{0}=a$ are square-free, coprimes, and that every prime number $p$ dividing $b+2 \eta j_{0}=c$ divides $b$. Every prime dividing $b+2 \eta j_{0}$ and $b$ must divide $2 j_{0}$, but if it divides $j_{0}$, then it will divide both $b$ and $a=b+\eta j_{0}$, which is impossible. Therefore, the only possibility is that either $c=b+2 \eta j_{0}=1$ or $j_{0}$ is odd, and that $b+2 \eta j_{0}$ is a power of 2 , say, $b+2 \eta j_{0}=2^{r}$ for some nonnegative integer $r$. This gives that $b=2^{r}-2 \eta j_{0}$ and that both $2^{r}-2 \eta j_{0}$ and $a=2^{r}-\eta j_{0}$ are positive and square-free. At any rate, note that in this case we have that $K=j_{0}^{2}$, therefore $|\gamma(m)-\gamma(n)|=|m-n|^{1 / 2}$, which confirms inequality (2.6) in this case as well. The remaining case, that is, the one for which $k-e j=0$, also does not lead to any solution by sign considerations.

The above arguments take care of part (i) of Theorem 2.3. Part (ii) can be proved in an identical manner as Theorem 2.1 (simply treat the number $j$ as a constant); note that since $|k|>j$, the degenerate instances considered above do not occur here. Finally, part (iii) of Theorem 2.3 follows from the above discussion of the degenerate instances. Theorem 2.3 is therefore proved.

5. Computational results. There exist 106 integers $r, 1<r<200$, such that $2^{r}-1$ and $2^{r-1}-1$ are both square-free. These are

$2,3,4,5,8,9,10,11,14,15,16,17,23,26,27,28,29,32,33,34$, $35,38,39,44,45,46,47,50,51,52,53,56,57,58,59,62,65,68$, $69,70,71,74,75,76,77,82,83,86,87,88,89,92,93,94,95,98$, 
TABLE 5.1

\begin{tabular}{|c|c|}
\hline$k$ & $n<10^{9}$ such that $\gamma(n+1)-\gamma(n)=k$ \\
\hline 3 & 4,49 \\
\hline 7 & 9,12 \\
\hline 11 & $20,27,288,675,71199$ \\
\hline 13 & $18,152,3024$ \\
\hline 15 & 16,28 \\
\hline 17 & $1681,59535,139239,505925$ \\
\hline 19 & $98,135,11375$ \\
\hline 21 & $25,2299,18490$ \\
\hline 23 & $75,1215,1647,2624$ \\
\hline 27 & 52,39325 \\
\hline 29 & $171,847,1616,4374$ \\
\hline 31 & $32,36,40,45,60,1375$ \\
\hline 39 & 76,775 \\
\hline 41 & 50,63000 \\
\hline 43 & 56,84 \\
\hline 45 & 22747,182182 \\
\hline 47 & $92,1444,250624$ \\
\hline 49 & $54,584,21375,23762,71874,177182720$ \\
\hline 53 & $147,315,9152,52479$ \\
\hline 55 & $512,9408,12167,129311$ \\
\hline 59 & 324,4239 \\
\hline 67 & $72,88,132,5576255$ \\
\hline 69 & 82075,656914 \\
\hline 71 & $140,3509,114375$ \\
\hline 73 & $872,1274,3249$ \\
\hline 75 & $148,105412,843637$ \\
\hline 79 & $81,104,117,156,343,375,7100,47375,76895$ \\
\hline 83 & $164,275,5967,33124,89375,7870625,38850559$ \\
\hline 85 & $126,1016,16128,471968,10028976$ \\
\hline 89 & 531,11736 \\
\hline 91 & $96,100,1050624$ \\
\hline 93 & $832,201019,1608574$ \\
\hline 97 & $3807,4067,12716,73304$ \\
\hline 99 & $112,1975,8575$ \\
\hline
\end{tabular}


TABLE 5.2

\begin{tabular}{r|l}
\hline \multicolumn{1}{c|}{$k$} & $n<10^{9}$ such that $\gamma(n+1)-\gamma(n)=-k$ \\
\hline 5 & 7,11 \\
7 & 44,80 \\
9 & 19 \\
11 & $17,360,31212$ \\
13 & $15,175,944,69375$ \\
17 & $23,351,1183,5750,240064$ \\
19 & $63,116,120,242,29645$ \\
21 & 43,424 \\
23 & $26,99,279,2400,110079$ \\
25 & $51,1808,2808$ \\
27 & 1519 \\
29 & $31,35,39,59,168,2375,6655,167112000$ \\
31 & 350 \\
33 & 67 \\
35 & 423,1376 \\
37 & 9800 \\
41 & $47,55,62,83,296,824,3699,3968,100499$ \\
43 & $207,260,528,5687$ \\
45 & 91 \\
47 & 53,539 \\
49 & $1475,3536,317600,834272$ \\
51 & 9250 \\
55 & 332 \\
57 & 115,124 \\
59 & $74,89,711,735$ \\
61 & $123,62000,945624$ \\
65 & $71,87,131$ \\
67 & $1224,11583,362556$ \\
69 & $79,139,18784$ \\
71 & 855,2988 \\
73 & $188,549,624,783,975,2645,28593$ \\
77 & $103,155,1368,129032$ \\
79 & $476,725,2600,2783$ \\
81 & 163,10624 \\
83 & $97,4655,26568,334719$ \\
85 & 6128 \\
87 & 244 \\
89 & $95,119,440,58080,1292400$ \\
91 & $548,1025,2208,50255$ \\
93 & 187 \\
95 & 1143 \\
97 & $111,6992,44375,68607$ \\
\hline & \\
\hline 5
\end{tabular}


$99,104,107,112,113,116,117,118,119,122,123,124,125,128$, $129,130,131,134,135,142,143,146,149,152,153,154,158,159$, $164,165,166,167,170,171,172,173,176,177,178,179,182,183$, 184, 185, 188, 191, 194, 195, 196, 197.

There exist 113 integers $r, 1<r<200$, such that $2^{r}+1$ and $2^{r-1}+1$ are both square-free. These are

$5,6,7,8,12,13,14,17,18,19,20,23,24,25,26,29,32,35,36,37$, $38,41,42,43,44,47,48,49,53,54,59,60,61,62,65,66,67,72$, $73,74,77,80,83,84,85,86,89,92,95,96,97,98,101,102,103$, $104,107,108,109,113,114,115,116,119,120,121,122,125,126$, $127,128,132,133,134,137,138,139,140,143,144,145,146,149$, $152,155,156,157,158,161,162,163,164,167,168,169,173,174$, $175,176,179,180,181,185,186,187,188,192,193,194,197,198$, 199, 200.

Moreover, here Table 5.1 presents all the solutions $n<10^{9}$ to the equation $\gamma(n+1)-\gamma(n)=k$, for $1<k<100$ (note that this equation has no solution $n<10^{9}$ for $k=5,9,25,33,35,37,51,57,61,63,65,77,81,87$, and 95.)

Finally, Table 5.2 presents all the solutions $n<10^{9}$ to the equation $\gamma(n+$ $1)-\gamma(n)=-k$, for $1<k<100$ (note that this equation has no solution $n<10^{9}$ for $k=3,15,39,53,63,75$, and 99).

ACKNOWLEDGMENTS. The authors would like to thank the referees for their valuable comments which helped improve the quality of this note. The first author was partially supported by a grant from NSERC, while the second author was partially supported by Grant SEP-CONACyT 37259-E.

\section{REFERENCES}

[1] R. Balasubramanian, M. Langevin, T. N. Shorey, and M. Waldschmidt, On the maximal length of two sequences of integers in arithmetic progressions with the same prime divisors, Monatsh. Math. 121 (1996), no. 4, 295-307.

[2] T. Cochrane and R. E. Dressler, Gaps between integers with the same prime factors, Math. Comp. 68 (1999), no. 225, 395-401.

[3] P. Cutter, A. Granville, and T. J. Tucker, The number of fields generated by the square root of values of a given polynomial, Canad. Math. Bull. 46 (2003), no. $1,71-79$.

[4] P. Erdôs and L. Mirsky, The distribution of values of the divisor function $d(n)$, Proc. London Math. Soc. (3) 2 (1952), 257-271.

[5] P. Erdős, C. Pomerance, and A. Sárközy, On locally repeated values of certain arithmetic functions. II, Acta Math. Hungar. 49 (1987), no. 1-2, 251-259.

[6] _ On locally repeated values of certain arithmetic functions. III, Proc. Amer. Math. Soc. 101 (1987), no. 1, 1-7.

[7] A. Granville, $A B C$ allows us to count squarefrees, Internat. Math. Res. Notices (1998), no. 19, 991-1009.

[8] D. R. Heath-Brown, The divisor function at consecutive integers, Mathematika 31 (1984), no. 1, 141-149.

[9] A. Hildebrand, The divisor function at consecutive integers, Pacific J. Math. 129 (1987), no. 2, 307-319. 
[10] M. Langevin, Partie sans facteur carré d'un produit d'entiers voisins [Squarefree part of a product of neighboring integers], Approximations Diophantiennes et Nombres Transcendants (Luminy, 1990), de Gruyter, Berlin, 1992, pp. 203-214 (French).

[11] __ Sur quelques conséquences de la conjecture $(a b c)$ en arithmétique et en logique, Rocky Mountain J. Math. 26 (1996), no. 3, 1031-1042 (French).

[12] D. S. Mitrinović, J. Sándor, and B. Crstici, Handbook of Number Theory, Mathematics and its Applications, vol. 351, Kluwer Academic Publishers Group, Dordrecht, 1996.

Jean-Marie De Koninck: Département de Mathématiques, Université Laval, Québec, Canada G1K 7P4

E-mail address: jmdk@mat.u1ava 1.ca

Florian Luca: Mathematical Institute, University Nacional Autónoma de México (UNAM), Apartado Postal 61-3 (Xangari), CP 58 089, Morelia, Michoacán, Mexico

E-mail address: fluca@matmor.unam.mx 


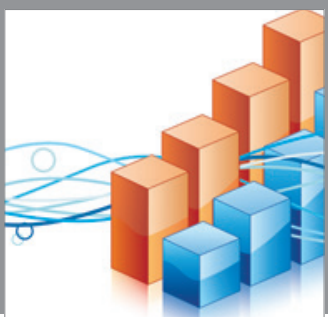

Advances in

Operations Research

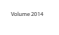

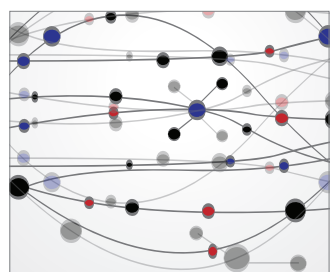

\section{The Scientific} World Journal
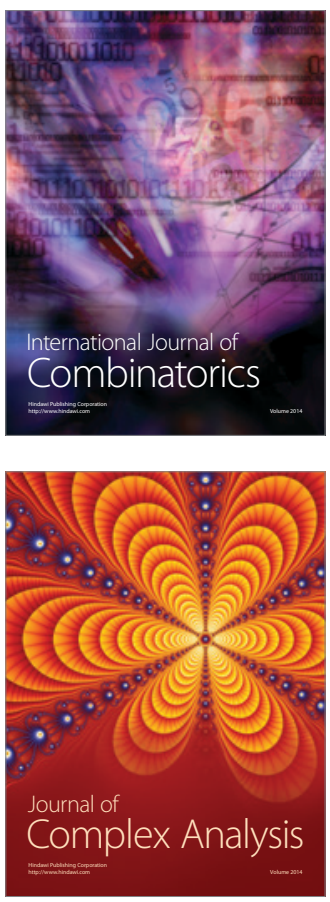

International Journal of

Mathematics and

Mathematical

Sciences
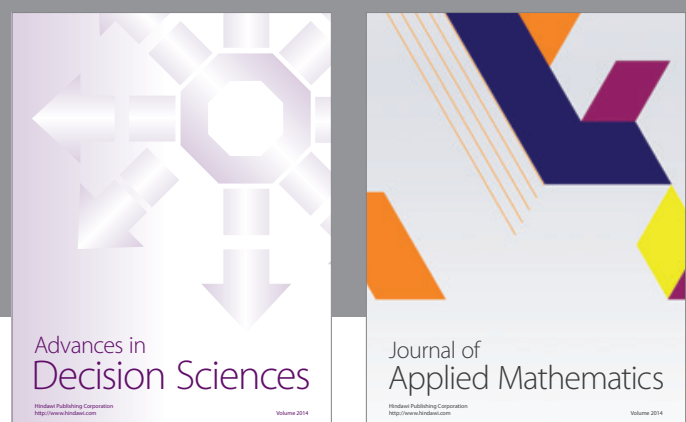

Journal of

Applied Mathematics
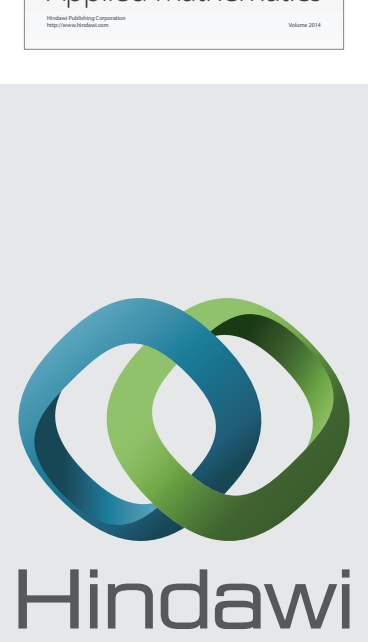

Submit your manuscripts at http://www.hindawi.com
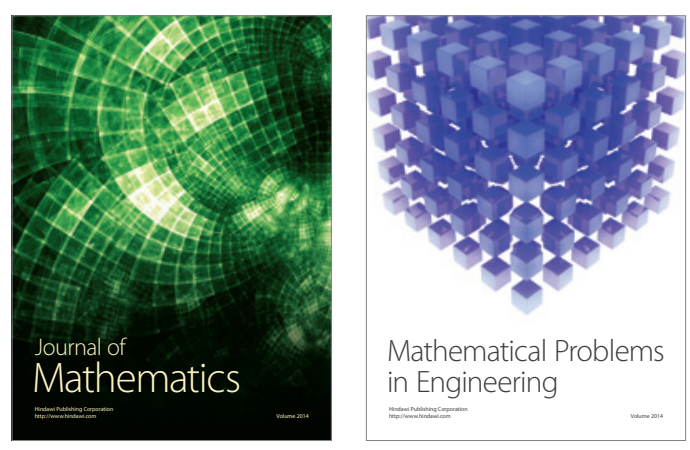

Mathematical Problems in Engineering
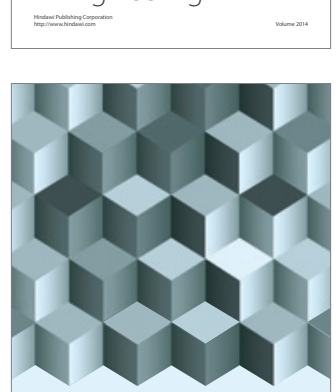

Journal of

Function Spaces
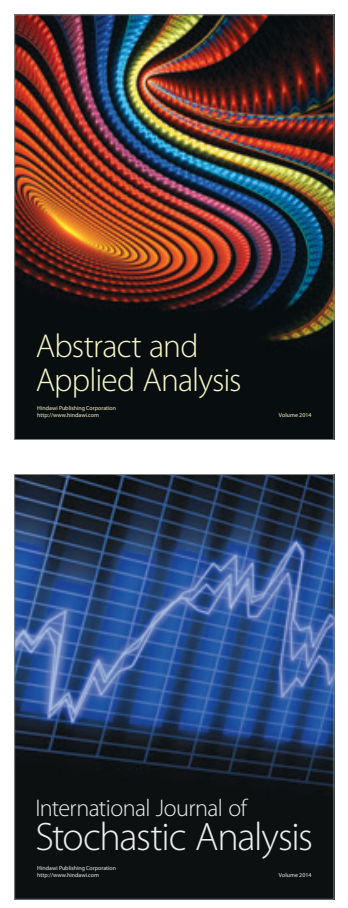

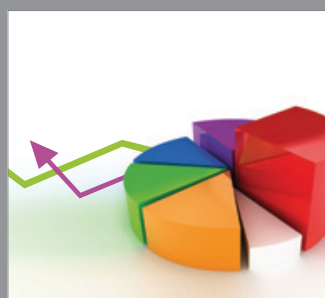

ournal of

Probability and Statistics

Promensencen
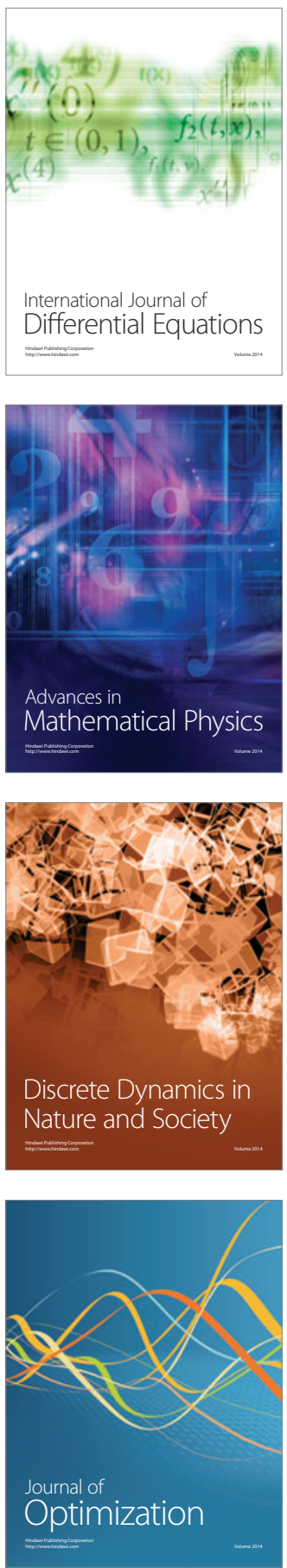